\title{
Advanced Qualification Methodology for Actively Cooled Plasma Facing Components
} \author{
Courtois ${ }^{1}$, J L Farjon ${ }^{1}$, M Missirlian ${ }^{1}$, J Schlosser ${ }^{1}$ and R Tivey ${ }^{2}$ \\ E-mail address: alain.durocher@cea.fr \\ ${ }^{1}$ Association Euratom-CEA, CEA/DSM/DRFC Cadarache, France \\ 2 IPP Garching, Germany \\ ${ }^{3}$ ITER Team, Cadarache, France
}

A Durocher ${ }^{1}$, F Escourbiac ${ }^{1}$, A Grosman ${ }^{1}$, J Boscary ${ }^{2}$, M Merola ${ }^{3}$, F Cismondi ${ }^{1}, \mathrm{X}$

\begin{abstract}
The use of high heat flux plasma facing components in steady state fusion devices requires high reliability. These components have to withstand heat fluxes in the range of 10-20 $\mathrm{MW} / \mathrm{m}^{2}$ involving a number of severe engineering constraints. Experience feed back of various industrial manufacturing showed that the bonding of the refractory armour material onto the metallic heat sink causes generic difficulties strongly depending on materials qualities and specific design. As heat exhaust capability and lifetime of plasma facing components during plasma operation are directly linked to the manufacturing quality, a set of qualification activities was performed during the component development phases following a qualification route. The paper describes the major improvement stemming from better measurement accuracy and refined data processing and analyses recent developments aim at investigating the capability to qualify the component in situ during its lifetime.
\end{abstract}

\section{Introduction}

High Heat Flux plasma facing components (PFCs) in steady state fusion devices will have to reach very high performance such as accepting thermal fluxes as high as $20 \mathrm{MW} / \mathrm{m}^{2}$. They require in addition high reliability. This can be only guaranteed through a very high level of qualification obtained with a rigorous acceptance inspection protocol. To meet the PFC power exhaust requirements during plasma operation imposes the control of their thermal and mechanical integrity. PFCs are systematically controlled before their installation in fusion machine detecting and localising defects inside material and welded junctions and qualifying their capacity to be cooled. Such systematic acceptance tests obviously need tools for process development and qualification of critical parts. The major issue is to detect thermal discontinuities in the element, such as material flaws like cracks and debondings. These can cause hot spots on the component surface and may even lead to the destruction of the PFCs e.g. Critical Heat Flux (CHF) event. A set of qualification activities should be performed very early during the component development and subsequent manufacturing phases. The paper aims at pointing out the qualification route, which has been followed in order to define an acceptance criterion for flat tile target elements joining for the TORE SUPRA Toroidal Pumped Limiter (TPL) series production [1] and more recently for the WENDELSTEIN 7-X (W7-X) component preseries production [2]. Investigations for the International Thermonuclear Experimental Reactor (Iter) Divertor elements, which are more challenging due to the thicker armour material and the more complex monobloc geometry, are now prepared and will be done shortly (figure 1).

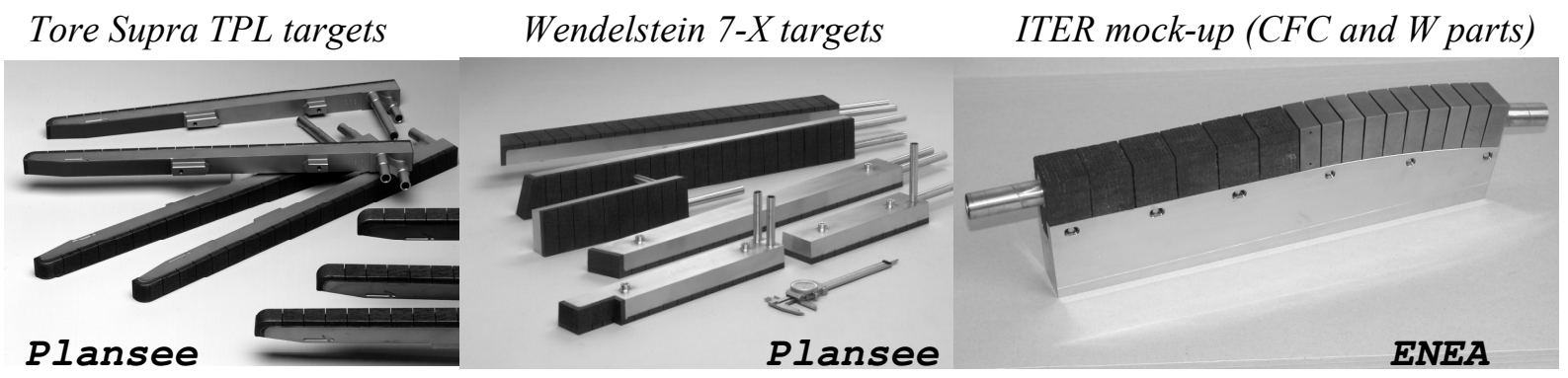

Figure 1: Various high heat flux plasma facing components. 
A long-term effort has been undertaken, from analysing the principles of the Non-Destructive Examination (NDE) techniques to their implementation at an industrial level. An assessment of the qualification methods was achieved, resulting in a more accurate qualification and in improvement of the NDE techniques. They now imply the data combination of various NDE. Recent developments aim at investigating the capability to qualify the component in-situ during its lifetime. They are summarized in the next sections.

\section{Plasma facing component challenge}

A plasma facing component designed to withstand heat fluxes in the range of $10-20 \mathrm{MW} / \mathrm{m}^{2}$ involves a number of severe engineering constraints: (i) the armour materials must be refractory and compatible with plasma wall interaction requirements; (ii) the heat sink should have a high thermal conductivity and high mechanical resistance; (iii) the cooling system, which is up to now based on a circulation of pressurized water in the PFCs heat sink, must offer a high thermal efficiency and margins to failure modes; (iv) the joint of the refractory armour material onto the metallic heat sink, which cause generic difficulties strongly depending on materials qualities and specific design, needs not to exhibit significant defects.

The ITER Divertor will be assembled with water actively cooled target elements, designed to withstand heat fluxes up to $20 \mathrm{MW} / \mathrm{m}^{2}$ [3]. As for the TORE SUPRA limiter and W7-X Divertor, the armour material - either 3D carbon reinforced carbon composite (CFC) or W - will be attached via a soft copper layer to a $\mathrm{CuCrZr}$ heat sink. Unfortunately any the joining methods severely damage the joints as it involves a high amplitude thermal cycle where the internal thermal stresses may reach high value in view of the very different thermal expansion of the materials at stake.

From the design, qualification prototypes are manufactured at various scales, small, medium and full scale. First of all, this phase allows to validate the fabrication feasibility before the study of the PFCs thermal mechanical behaviour. The main goal of this R\&D phase is to assess the thermo mechanical behaviour of component under cycling high thermal flux. The initial and final NDE, such as active infrared thermography, will allow to assess the cooling capability of the PFCs.

For TORE SUPRA TPL manufacturing, thermal fatigue tests at $10 \mathrm{MW} / \mathrm{m}^{2}$ were performed at FE200 (electrons beam facility 200KW- FRAMATOME Le Creusot-F) and showed a significant increase of surface temperature after 2000 cycles with an uncertainty zone between 2000 and 3000 cycles. Observations showed that degradation were appeared well over 3000 cycles (figure 2). This study allowed roughly to define a steady state surface temperature limit of $1200^{\circ} \mathrm{C}$ at $10 \mathrm{MW} / \mathrm{m}^{2}$ for $\mathrm{TPL}$ targets [4]. The same study is being performed for W7-X Divertor and ITER Divertor vertical targets.

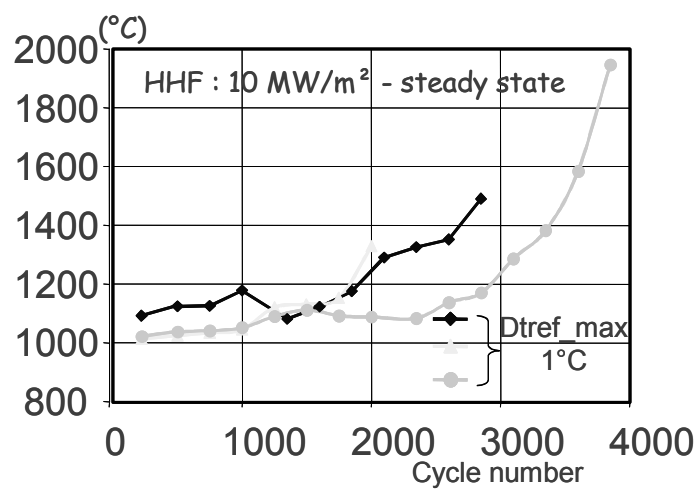

Figure 2: Fatigue ageing - Evolution of surface temperature (TPL).

The ability to predict the evolution of flaws assembling need a thermal fatigue analysis which is a challenging problem including both experimental activities based on development of dedicated NDE, such as active infrared thermography technique or High Heat Flux (HHF) testing and thermo mechanical modelling activities. If the issue of fatigue damage is extremely difficult especially when it involves composite element such as CFC, pure metal and hardened alloys such as $\mathrm{CuCrZr}$, it seems reasonable to first focus on possible defects produced during the manufacturing process. In fact any 
flaw will have a tendency to spread under further stresses in operation. Hence the necessity is to detect even minor defects at this stage. Consequently the development of functional NDE, like active infrared thermography using the heat transfer method, appeared very early as a necessity as high heat flux testing is extremely time and cost consuming. It was possible to assess the capability of the so-called SATIR test (French acronym for Infra Red Acquisition and Data Processing device) even during this development period [5]. Even though it appeared to be an excellent tool from the beginning, the methodology had to be improved so as to reach a good reliability. This paper will not address many other issues impacting the plasma facing component design. However, in any case, the qualification methodology may help in minimizing the risks associated to the component throughout the design optimisation and series acceptance and then in assessing the possible failure modes of the component if the mechanical and thermal defects are detected.

\section{Qualification methodology}

\subsection{The SATIR test and its limits}

The SATIR test is based on the detection of a delay in the surface temperature evolution produced through an abrupt variation of the water temperature flowing in the cooling tube [6]. This delay is actually measured in comparison to the behaviour of a reference element. This is realized by an alternate flow of cold $\left(5^{\circ} \mathrm{C}\right)$ and hot $\left(95^{\circ} \mathrm{C}\right)$ water into the cooling channels (figure 3$)$. The principle is based on the comparison of the surface temperature evolution of the inspected component with that of a so-called "defect-free" reference one. In the case of the TPL, two elements were tested in parallel to a reference and a DTref criterion (Maximum of the transient temperature difference: figure 4) was stated to $3^{\circ} \mathrm{C}$ maximum mismatch with respect to this reference. The connection between this mismatch and defect size was established both by means of finite element modelling, taking into account material physical properties variation, thickness effect and the background noise of the facility, and by tests of calibrated defects.

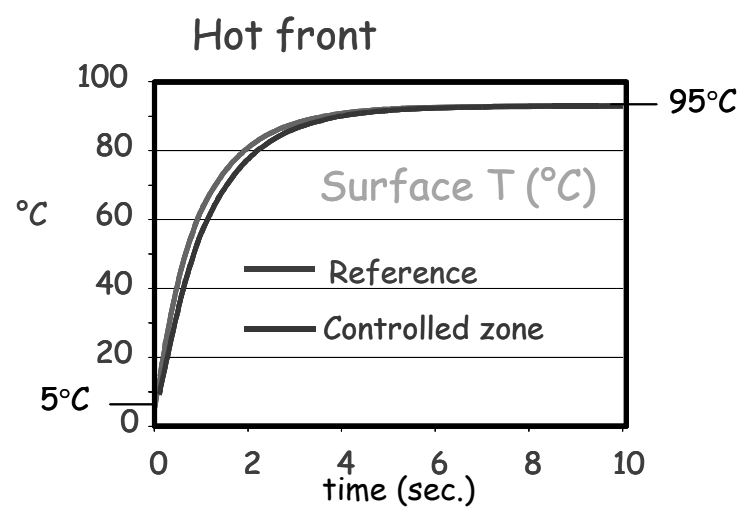

Figure 3: Surface temperature evolution: tested and reference element.

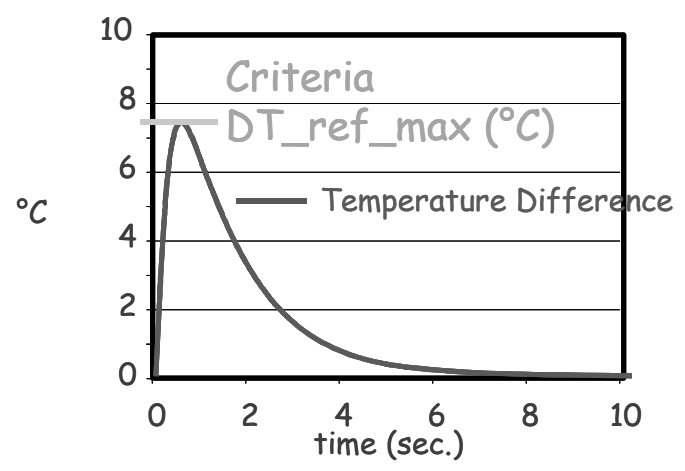

Figure 4: DTref_max definition (data similar as in figure 3 ).

Considering the SATIR performances during TPL commissioning, it was considered that corner interface defects larger than $6 \mathrm{~mm}$ required detection with confidence. The mismatch extent was derived from the uncertainties in the material characteristics and the measurement $( \pm 0.5 \mathrm{~K}$ for $\pm 5 \%$ on the $\mathrm{CFC}$ conductivity, $0.1 \mathrm{~K}$ for $\pm 1 \mathrm{~mm}$ on the CFC thickness, $\pm 2.1 \mathrm{~K}$ for $\pm 0.7 \mathrm{~mm}$ on the copper thickness, $0.2 \mathrm{~K}$ for IR camera). A micrographic examination allowed to quantify the correlation of the SATIR DTref value to free cracks extent. This finally gave access to the minimum value detectable. Heat transfer degradation has been efficiently assessed (figure 5). As results from SATIR gave evidence of defective tiles in good correlation with High Heat Flux (HHF) tests. Such value must be lower than the maximum acceptable value defined by design conditions. It appeared that the margin although acceptable needed improvement to meet the challenge of very large amount: even for the TPL, the number of tiles exceeded 12000 and in a few cases elements not meeting the criterion 
requirements were accepted and installed in the Tokamak, taking the advantage that not all the PFCs are subjected to the maximum heat exhaust design value [5].

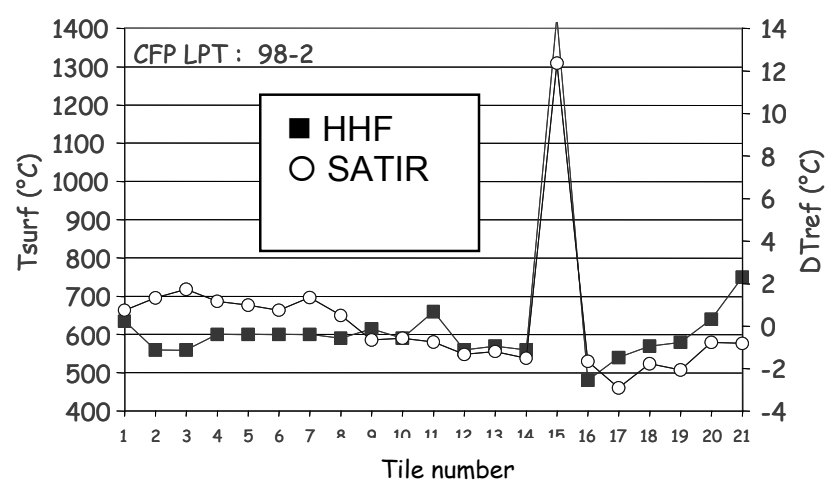

Figure 5: SATIR and High Heat Flux (HHF) tests correlation (TPL application).

\subsection{Improvement of the SATIR test}

In order to improve the defect detection capability of the SATIR test bed, several possibilities have been considered and assessed and some of them already implemented. In particular the installation in 2003 of a digital infrared camera and the improvement of the thermal signal processing led to a considerable improvement of the performances. The emissivity correction of the measured surface took an important part in this improvement, a pixel normalization algorithm has been developed and allowed the detect threshold to be reduced. In fact both the normalization and the DTref by pixel allowed to increase the spatial localization and detection sensitivity by a factor 2 . In the case of the TPL element, corner flaw of $3 \mathrm{~mm}$ and strip flaw of $2 \mathrm{~mm}$ can be now easily detected at the $\mathrm{CFC} / \mathrm{Cu}$ bonding (figure 6).

\section{Corner flaw $(3 \mathrm{~mm}) ;(6 \mathrm{~mm})$}

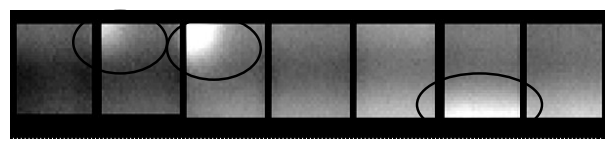

Strip flaw (2mm)
Figure 6: SATIR DTref_max cartography on

TPL target with calibrated defects

(CFC flat tile - CFC thickness of 6mm).

The advanced data processing was highlighted on SATIR DTref_max image of a CFC baffle panel from ITER monobloc technology and image of the same tested component exposed to high heat flux of $5 \mathrm{MW} / \mathrm{m}^{2}$. Similar default patterns (shown by a black circle) are observed with both techniques (figure 7). A correct agreement between SATIR infrared examination and high heat flux testing allowed to validate the active infrared thermography technique as a functional ITER relevant method.

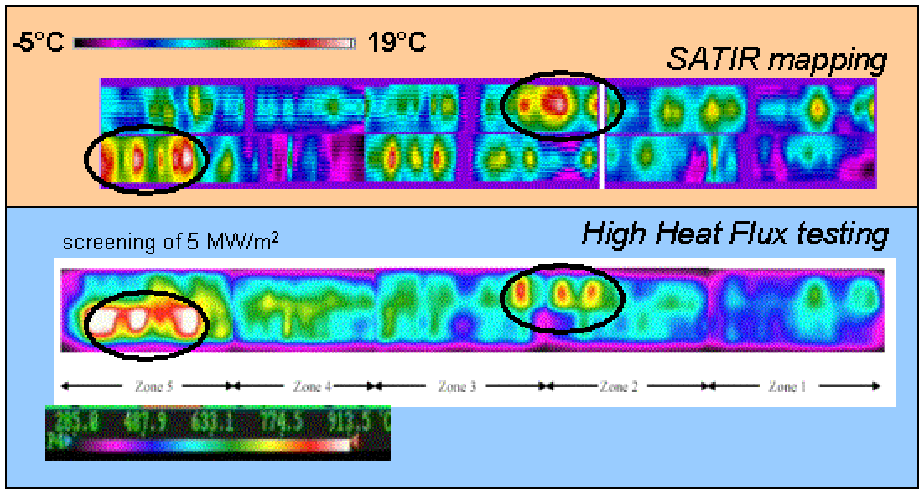

Figure 7: Good agreement between SATIR infrared image and high heat flux testing (CFC monobloc). 
However, to reduce the uncertainty measurements due to the use of reference element some developments were performed using signal processing methods. After a spatial-temporal filtering of the signal, a data process based on spatial image autocorrelation allows a better localization of the bond defect. The qualitative improvement of the defect detection obtained by signal processing methods is combined with a quantitative estimation accomplished by analytical simulation of the thermal behaviour of a component tested on SATIR test bed. This method using a digital reference allows to avoid the reference element. In addition, a 1D model based on thermal quadruples theory, allows to quantify the defect in terms of thermal contact resistance [7]. This methodology has been applied to the qualification tests of W7-X PFCs pre series (figure 8) and will be soon apply to the ITER monoblocs.
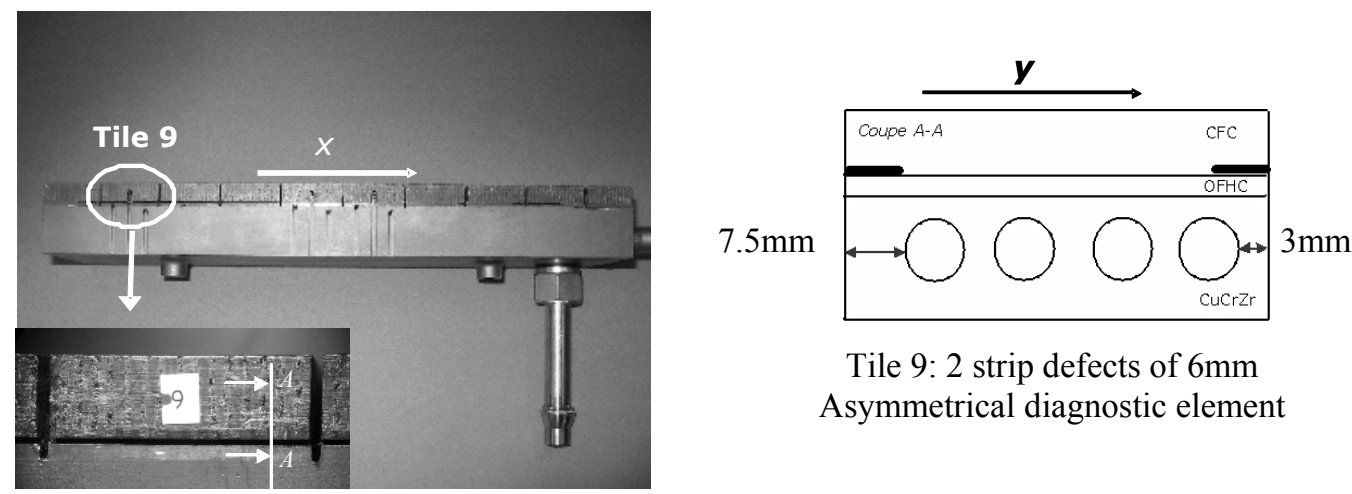

Tile 9: 2 strip defects of $6 \mathrm{~mm}$ Asymmetrical diagnostic element

Figure 8: W7-X preserie target with calibrated defects (asymmetrical geometry).

The figure 9 shows the analysis of faulty tile \#9 (2 artificial $6 \mathrm{~mm}$ strip flaw) of an asymmetrical diagnostic element. Image processing and analytical model yield complementary information on defect detection and estimation. The CFC thickness of W7-X PFCs is $8 \mathrm{~mm}$.

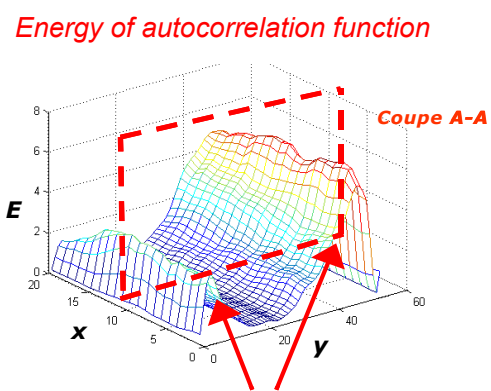

$6 \mathrm{~mm}$ strip defects

Free defects element: Energy $<0.1$

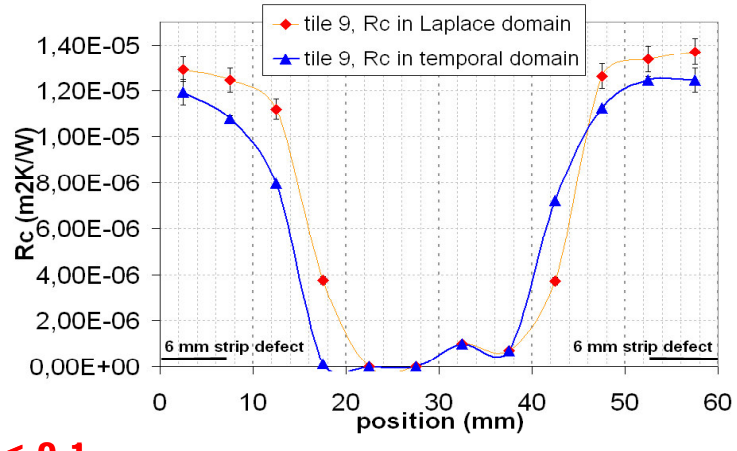

Figure 9: Energy of autocorrelation function and estimation of the

Thermal contact resistance (Rc). A digital reference replaces the element reference.

\subsection{Application of multiple NDE techniques for recent projects such as $W 7-X$}

The Divertor of the Stellarator W7-X is composed of 890 actively cooled target elements with a total area of $19 \mathrm{~m}^{2}$, and was designed to withstand heat fluxes up to $10 \mathrm{MW} / \mathrm{m}^{2}$ in steady state operation. The selection of the material combination of the target elements is a 3D carbon fibre reinforced carbon composite (CFC grade Sepcarb® NB31), joined to a heat sink of CuCrZr alloy. The definition of acceptance criteria is based on manufacturing and qualification experiences obtained during a pre series fabrication representing about 30 full-scale elements [8]. A crosschecking analysis, between the high beat flux performance tests (mainly carried out in the ion beam facility GLADIS [9] at IPPGarching), and the non-destructive infrared thermography examination has been performed. It allowed to set a DTref_max detectable limit for SATIR close to $1.5^{\circ} \mathrm{C}$ as well as to define an acceptance limit 
of about $4^{\circ} \mathrm{C}$ for the standard elements, corresponding to a surface temperature increase of about $30 \%$ with respect to the surface average temperature under a heat flux of $10 \mathrm{MW} / \mathrm{m}^{2}$ (figure 10). The newly defined acceptance criteria for the flat tiles target elements may apply for reception of the W7-X Divertor targets during series fabrication [10].

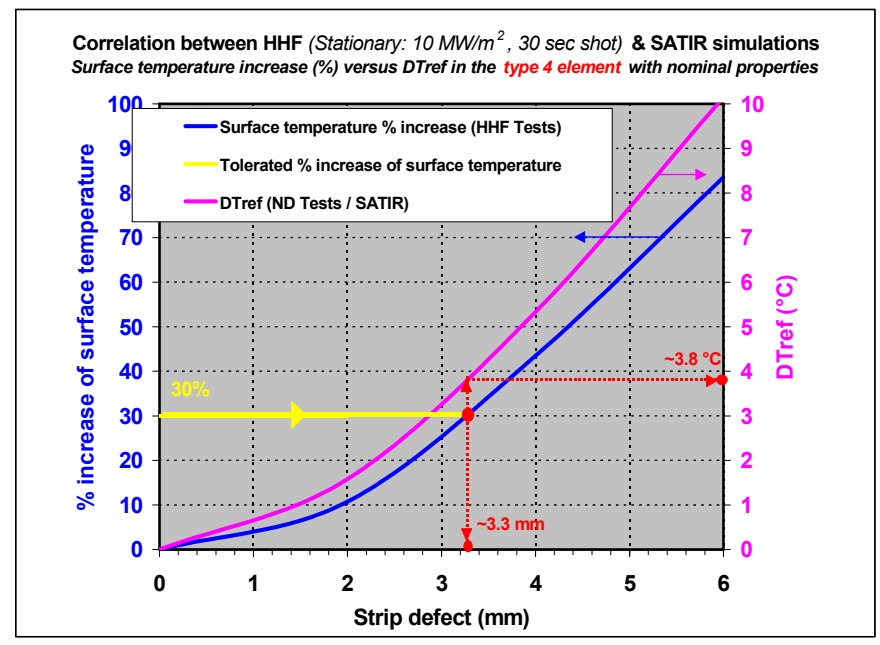

Figure 10: Acceptance criteria evaluation for standard W7-X target, from the increase of the surface temperature as function of the defect size.

To avoid taking decisions, which are doubtful when in particular, the threshold is only slightly exceeded; it appeared that the use of a second technique probing again the thermal response of the component might help. Modulated photothermal thermography and lock-in detection (to be summarized hereafter as "LOCKIN") consists of the exposure of the component tile surface by a periodic external thermal source while recording its temperature response by infrared thermal imaging, and then calculating the phase shift between the measured signal and the source by synchronous demodulation (figure 11)[11]. The phase-shift of the thermal response depends on the thermal conductivity and diffusivity along the heat path, thus on the presence of any thermal discontinuity within the material, leading to a localized modification of diffusivity. This allows to detect strong thermal resistances such as produced by cracks or debonding, provided they are large enough compared to the distance to the temperature measurement surface so as to maintain the 1D character of the analysis modelling. Note that other thermal stimulation (e.g. pulsed) can be used and have been implemented. In any case, the definition of criterion will be limited by the rather poor statistics that one can derive from results obtained in pre series where about 100 elements might be tested and submitted to high heat flux tests. Nevertheless, criteria may be guessed defining the boundary of a zone of confidence to a zone of semi confidence and of the latter to a zone of non-confidence.

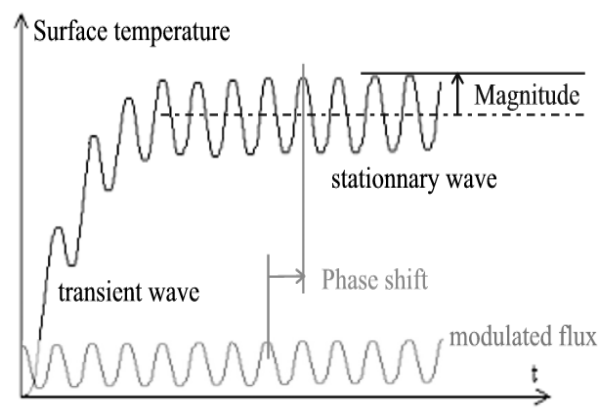

Figure 11: Sinusoidal thermal stimulation: LOCKIN Thermography showing the modulated flux and the surface temperature evolution. 


\subsection{Data merging}

Data merging from the above quoted techniques appeared to improve significantly the detection sensibility and reliability of defect location and size. This original study, using fuzzy logic through a Dempster-Shafer's model, showed a detection improvement of LOCKIN and SATIR methods (figure 12). The data merging method is now in operation at CEA [12]. From the experimental know-how gained during the development of each individual NDE method, it will be possible to implement a data merging process with fairly reduced training. This data process brings a significant improvement even for a method that is already very reliable like the SATIR method. In the future the merging of additional NDE on ITER monoblocs, like the ultrasonic inspection or low heat flux examination data should improve the reliability of the acceptance.

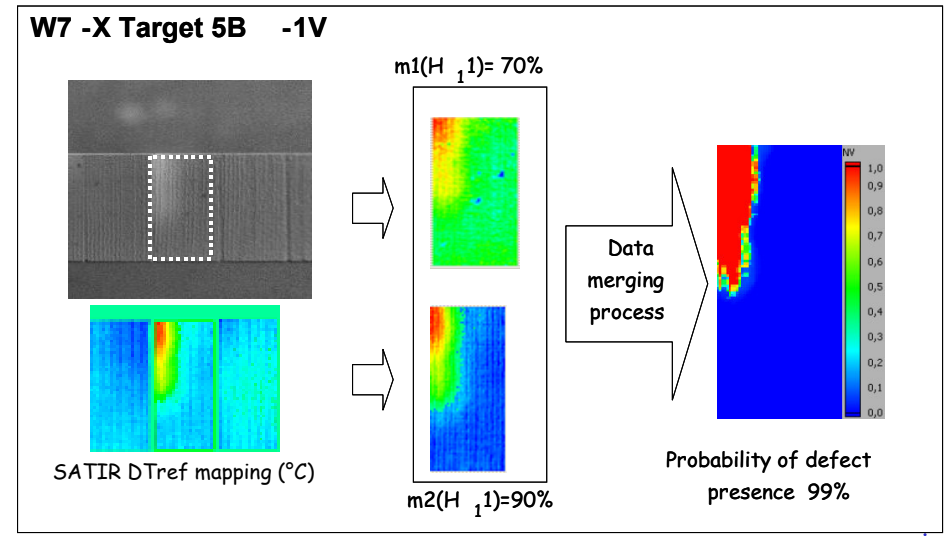

Figure 12: Infrared data merging (SATIR and LOCKIN) on a faulty W 7-X element tile. The $\mathrm{m}_{\mathrm{i}}(\mathrm{H} 1)$ value represents the probability of defect presence for the i NDE

(LOCKIN $i=1$; SATIR $i=2$ ).

\subsection{ITER prospects}

SATIR inspection is proposed as the basis test to decide upon the final acceptance of the ITER Divertor qualification prototypes. So as to provide a statistical basis, an extensive study is undertaken under EFDA auspices for the ITER Divertor vertical target composed by 112 samples of W and CFC armoured PFCs with calibrated defects located at the interfaces are being manufactured in Europe both for advanced NDE validation and HHF testing [13].

\section{Operational assessment of the PFCs}

A growing requirement is to try to investigate the operational modes of the component including the failure modes in view of the limitation of the ITER PFCs and also to check the capability to assess the component thermal and mechanical status in situ. An investigation of the CFC erosion is presented hereafter.

\subsection{Investigation on the erosion phenomenon}

In the case of ITER Divertor, the CFC monobloc in the vertical target is the most loaded part of the plasma facing surfaces, hence a significant risk of failure; if the monobloc is expected to retain the CFC armour around the tube even after debonding, the possibility of a strong erosion could happen and should be assessed. A program has been developed with the aim of understanding the impact on the erosion lifetime and on the probability of a critical heat flux event in the heat sink of a combination of two main effects: the material property variations and the presence of joining defects. Within assessing erosion, the finite element (FE) calculation takes account of geometry and sublimation, physical and chemical erosion of the CFC armour (figure 13). The programs have been validated by comparison with analytical and experimental data. 


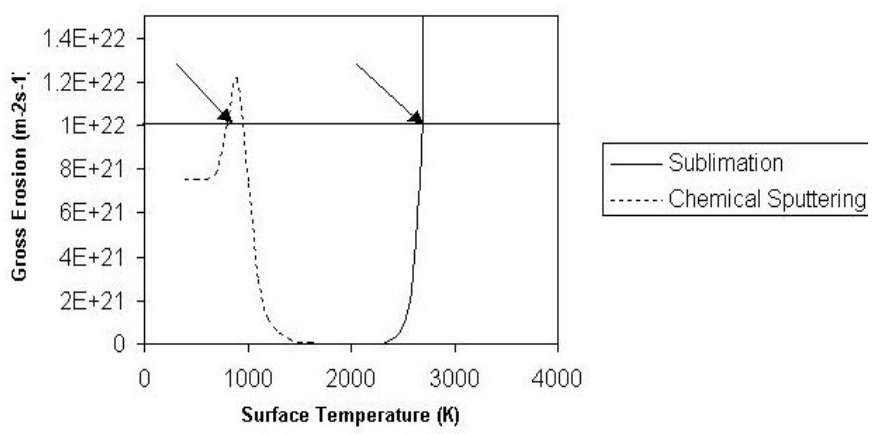

Figure 13: Gross erosion flux as function of surface temperature for detached plasma case.

The figure 14 shows a ANSYS calculation of physical erosion at $10 \mathrm{MW} / \mathrm{m}^{2}$ performed on nominal ITER monobloc geometry with calibrated interface defects. To simulate the erosion progress, a refined mesh has been used up to $2 \mathrm{~mm}$ under the top surface of the components. Under these conditions the surface temperature reached to $2250^{\circ} \mathrm{C}$ [14]. In the next step, the model shall be achieved by implementing the various phenomena and will help in modelling the macroscopic behaviour of a Divertor element and especially check the eventuality of catastrophic failure such as critical flux event or strong reduction of the component lifetime by CFC ablation.

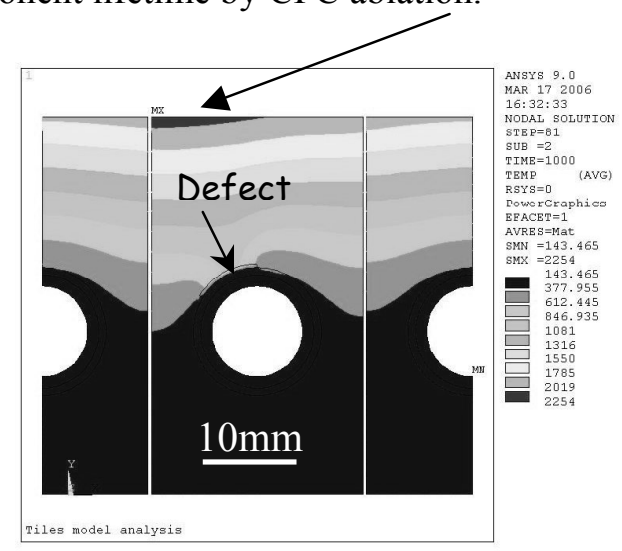

Figure 14: 2D ANSYS calculation of physical erosion $\left(\phi 0=1023 \mathrm{D} / \mathrm{m}^{2} / \mathrm{s} ; \mathrm{E}=30 \mathrm{eV}\right)$ at $10 \mathrm{MW} / \mathrm{m}^{2}$ on ITER monobloc with a calibrated defect.

\subsection{In situ monitoring of PFCs}

Because it is difficult to prevent the creation or evolution of defects, it is essential to relate the quality of the component to its possible evolution in operation. For instance, non-destructive testing devices as the lock-in thermography technique - may be applied to observe, in between plasma campaigns, the evolution of defects in the TORE SUPRA TPL CFC/Cu joints. In the case of TPL elements, the quality of the elements was controlled on $100 \%$ of the delivered batches. In order to integrate the TPL, a new relaxed acceptance criterion for flat tiles was applied to delivered elements. Some elements, with tiles outside the criteria range, but accepted nevertheless, were installed on the limiter sectors with lower power densities taking into account the shadowing on the TPL due to the Shafranov shift and the ripple effect. These shadowed zones have been experimentally confirmed during the 2001 campaign as they exhibited strong redeposition amount of eroded carbon (figure 15) [15]. 


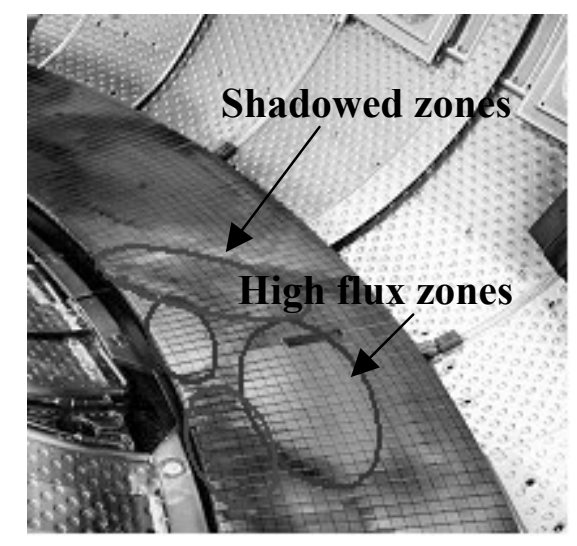

Figure 15: Shadowed and flux zones on TORE SUPRA TPL

A LOCKIN device has been implemented in situ (figure 16) during plasma shutdown through an external sinusoidal thermal excitation generated by a set of modulated halogen lamps. The thermal response of the inspected component is recorded during the stimulation with the help of an infrared device. Magnitude and phase-shift of the response, which is calculated with a synchronous demodulation, may indicate the presence of cracks failure into the component (phase-shift map), but also the presence of coatings due to co deposition phenomena of plasma component mainly $\mathrm{C}$ and $\mathrm{D}$ (magnitude map).

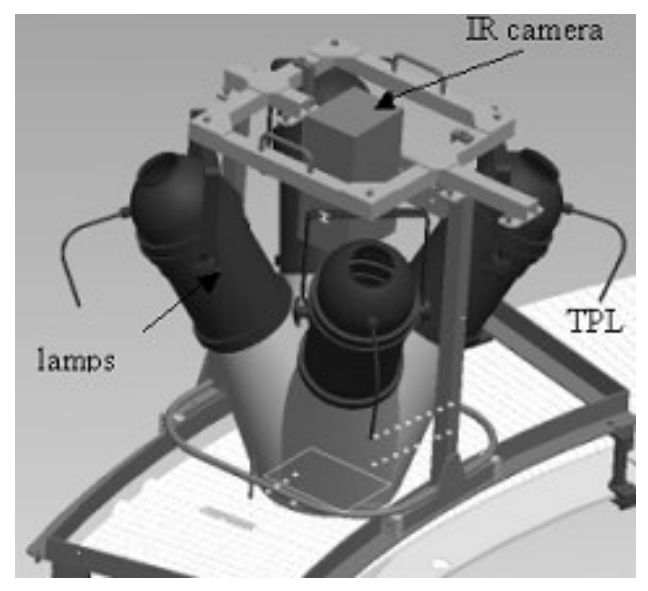

Figure 16: LOCKIN test device set up on TPL in Tore Supra vacuum vessel

(Electronics and computer are set-up outside the vessel)

Reliable measurements of magnitude and phase-shift contrast were obtained on a limiter element with calibrated flaws machined at the bonding $\mathrm{CFC} / \mathrm{Cu}[16]$. The resulting cartography of a TPL sector shows 2 tiles at least with probable reduced heat transfer efficiency (figure 17). The results are still preliminary and may suffer from the non-appearance of defective tiles as the TPL is still used below its thermal heat exhaust design values. 


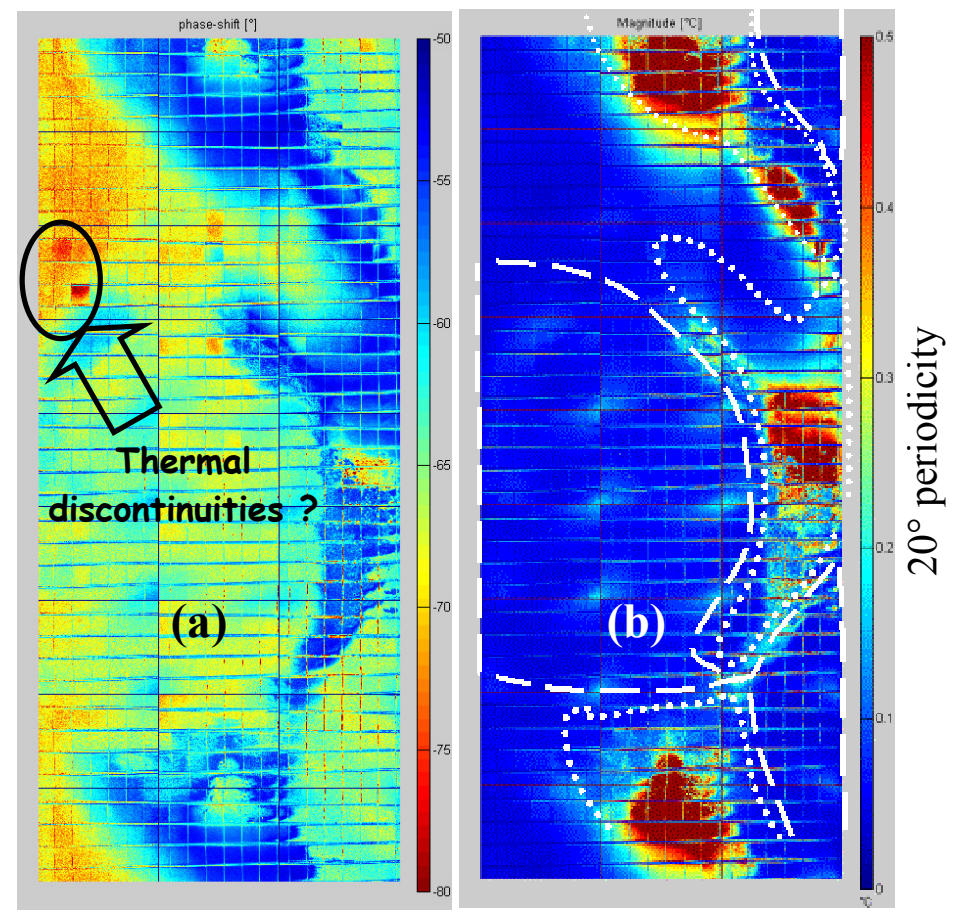

Figure 17: Phase-shift (a) and magnitude (b) LOCKIN maps of TPL Q6A sector

Dotted lines $=$ max carbon deposit areas, dashed lines $=$ erosion area

\subsection{Acoustic precursor of critical heat flux for safety monitoring}

First experiments were performed during HHF testing at FE200 facility on a tungsten armoured hypervapotron component during an experiment on CHF. The mock-up equipped with piezoelectric accelerometers was heated by intense electron beam that simulates the high heat flux caused by a plasma discharge. The heat flux deposited on the mock-up was increased step by step up to the occurrence of critical heat flux, the step duration being $60 \mathrm{sec}$. During the last step, the absorbed heat flux was $32 \mathrm{MW} / \mathrm{m}^{2}$; the mock-up was destroyed after $41 \mathrm{sec}$. A fluctuation of the measured frequencies means that there are instabilities in the signal; this could be due to a transient regime of the physical phenomenon [16]. In other words it could possibly indicate the approach or the presence of a local CHF. The figure 18 represents the most visible frequency fluctuations found in the signal recorded before the rupture, which could provide a precursory indicator for online safety monitoring. There are a few others but they stand out less in the surrounding noise.

$$
<0>26>29>30>32>\mathrm{MW} / \mathrm{m}^{2}
$$

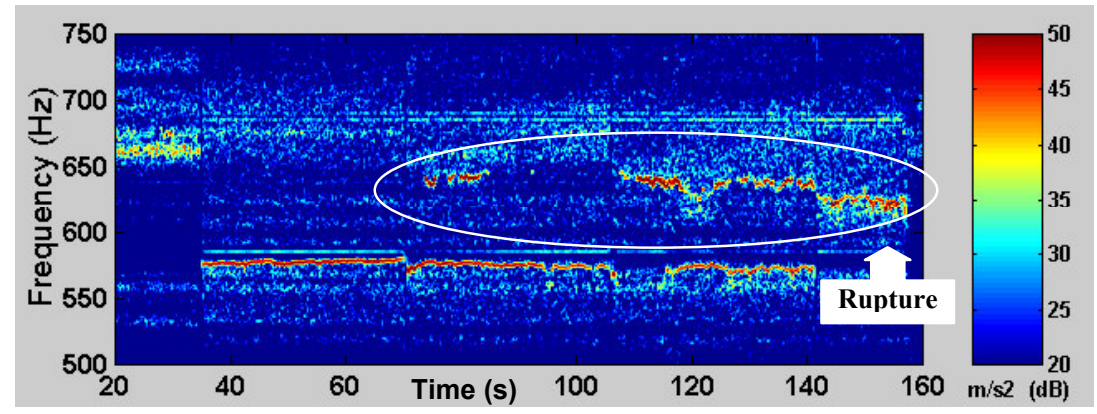

Figure 18: Frequency fluctuations recorded during successive increasing in the most power bursts by piezoelectric accelerometer 


\section{Conclusion}

Actively cooled plasma facing components sustaining high heat flux must be controlled following thermal and mechanical criteria. The major risk is that the heat loads can induce a partial or entire bond fracture between the refractory tile and the heat sink if any large cracks remain. Consequently, advanced non-destructive inspection and original in-situ monitoring techniques were developed for the qualification of PFCs including R\&D and the definition of acceptance criteria. This could be achieved by putting together various NDE methods and defining a methodology where the poor statistics may be used at its best by a strong effort on the modelling, data analysis and processing. The results are very encouraging in view of the ITER challenge. In-situ assessment of the PFCs is now investigated; the studies are still in a preliminary phase.

\section{Acknowledgments}

This work, supported by the European Communities under the contract of Association between EURATOM/CEA, was partially carried out within the framework of the European Fusion Development agreement. The views and opinions expressed herein don't necessarily reflect those of the European Commission.

\section{References}

[1] Schlosser J 2001 Experience feedback from high heat flux component manufacturing for Tore Supra Fusion Engineering and Design 56-57 309-13

[2] Boscary J 2005 Applied technologies and inspection for the W7-X pre-series target elements Fusion Engineering and Design 75-79 451-55

[3] Merola M 2005 EU R\&D on ITER Divertor components Fusion Engineering and Design 7579 325-31

[4] Cordier JJ 2003 Preliminary results and lessons learned from upgrading the Tore Supra actively cooled plasma facing components Fusion Engineering and Design 66-68 59-67

[5] Durocher A 2003 Quality control of plasma facing components for Tore Supra Fusion Engineering and Design 66-68 305-09

[6] Durocher A 2005 Development of an original active thermography method adapted to ITER plasma facing component control Fusion Engineering and Design 75-79 401-05

[7] Cismondi F 2005 Etude méthodologique des tests non destructifs des liaisons Composite à Fibre de Carbone-Cuivre par thermographie infrarouge Méthodes et techniques optiques pour l'industrie 6éme colloque international francophone (Marseille France)

[8] Missirlian M 2006 Infrared thermography inspection methods applied to the target elements of W7-X Divertor proc. of $24^{\text {th }}$ SOFT (Varsaw Poland)

[9] Greuner H 2005 Design, performance and construction of a 2 MW ion beam test facility for plasma facing components Fusion Engineering and Design 75-79 345-50

[10] Missirlian M 2006 Qualification of high heat flux components: application to target elements of Wendelstein 7-X, proc. of $11^{\text {th }}$ PFMC (Greisfwald Germany)

[11] Escourbiac F 2005 Application of Lock-in technique to CFC armoured plasma facing component inspection proc. of $12^{\text {th }}$ ICFRM (Santa Barbara USA)

[12] Durocher A 2006 Infrared images data merging for plasma facing component inspection proc. of $24^{\text {th }}$ SOFT (Varsaw Poland)

[13] Fouquet S 2006 Acceptance criteria for the ITER Divertor vertical target Fusion Engineering and Design $81265-68$

[14] D'Agata E 2003 Behaviour of the PFCs tiles on the ITER Divertor under critical loading conditions Fusion Engineering and Design 66-68 329-34

[15] Mitteau R 2002 Power exhaust and edge control in steady state TORE SUPRA plasma proc. of $19^{\text {th }}$ IAEA (Lyon France)

[16] Courtois X 2006 In-Situ Monitoring of Actively Cooled Plasma Facing Components Using Acoustic and Thermal Methods, proc. of $11^{\text {th }}$ PFMC (Greisfwald Germany) 